\title{
Monte Carlo simulation study of the high-temperature phase diagram of model $\mathrm{C}_{60}$ molecules
}

\author{
M. Hasegawa \\ Department of Materials Science and Technology, Faculty of Engineering, Iwate University, \\ Morioka 020-8551, Japan \\ K. Ohno \\ Institute for Materials Research, Tohoku University, Sendai 980-8577, Japan
}

(Received 17 May 1999; accepted 8 July 1999)

\begin{abstract}
The constant- $N V T$ Monte Carlo simulation is performed for model $\mathrm{C}_{60}$ molecules interacting via the Girifalco potential and a full free-energy analysis is made to predict the high-temperature phase diagram. The repulsive part of the $\mathrm{C}_{60}$ potential is very steep and the attractive part is relatively short-ranged. For such a system accurate computations of the virial pressure are difficult in simulations and it is argued that the discrepancies among the previous results for the phase diagram of $\mathrm{C}_{60}$ can partly be attributed to the uncertainties of the virial pressure involved in simulations. To avoid this difficulty we take the energy route to calculate equation of state (EOS), in which the absolute (Helmholtz) free energy is obtained by performing isochoric integration of the excess internal energy. A difficulty of the energy route in the high-temperature limit is resolved by the aid of an analytic method. The exact second and third virial coefficients are also used in the analysis of the fluid EOS. The pressure route is taken to calculate the EOS of the solid phase, in which the virial pressure is numerically more stable than in the fluid phase. The resulting high-temperature phase diagram of $\mathrm{C}_{60}$ is quite systematic and free from uncertainties, and the liquid-vapor critical point is found at $T_{c}=1980 \mathrm{~K}$ and $\rho_{c}=0.44 \mathrm{~nm}^{-3}$, whereas the triple point at $T_{t}=1880$ and $\rho_{t}$ $=0.74 \mathrm{~nm}^{-3}$, confirming the existence of a stable liquid phase over the range of $\sim 100 \mathrm{~K}$. $\odot 1999$ American Institute of Physics. [S0021-9606(99)51137-9]
\end{abstract}

\section{INTRODUCTION}

In an early theoretical work on colloidal suspensions, Gast $e t$ al. ${ }^{1}$ observed that for systems with sufficiently shortranged attractive potentials the sublimation line passes above the liquid-vapor critical temperature $T_{c}$ in the $T-\rho$ (temperature-density) plane, and hence the liquid phase is thermodynamically unstable. More recently, bulk $\mathrm{C}_{60}$ has attracted great attention as a possible candidate for such a substance ${ }^{2}$ and several simulations and theoretical calculations have been performed. Hagen $e^{a l .}{ }^{3}$ and Cheng et $a l^{4}$ were the first to have performed such simulations and reached different conclusions; the former used a Gibbsensemble Monte Carlo (GEMC) method and found that $\mathrm{C}_{60}$ has no liquid phase, whereas the latter used a molecular dynamic (MD) method and predicted the existence of a stable liquid phase between $\sim 1800 \mathrm{~K}$ and $\sim 1900 \mathrm{~K}$. Both of these authors used the same intermolecular potential proposed by Girifalco, ${ }^{5}$ but in the GEMC simulation of Hagen $e t$ al. this potential was truncated at $r=2 \sigma$, where $\sigma$ is the diameter of the $\mathrm{C}_{60}$ molecule. The effect of such a truncation was thought to be minute, but later theoretical investigation ${ }^{6}$ based on a density-function theory (DFT) of freezing revealed that the effect is substantial and the inclusion of the truncated part of the potential could lead to a different conclusion as to the existence of a stable liquid phase. We also note that the MD simulation results of Cheng $e t$ al. are subject to large uncertainties and quantitatively not very definite.

The GEMC method was also used in the simulation study of Caccamo et al. ${ }^{7}$ and the liquid-vapor critical point was found at $T_{c}=1920-1940 \mathrm{~K}$ and $\rho_{c}=0.39-0.42 \mathrm{~nm}^{-3}$ depending on the number of molecules in the simulation cell. They supplemented these simulation data by the freezing line from other sources and concluded that stable liquid phase exists in agreement with the MD results of Cheng et al. ${ }^{4}$ However, the location of the triple point estimated by Caccamo $e t$ al. was indefinite because the freezing line used in this estimation was phenomenological or subject to large uncertainty.

Broughton $e t$ al. ${ }^{8}$ took a somewhat different approach in their MD simulation study. They employed a shell model in which the radius of each $\mathrm{C}_{60}$ molecule was allowed to be a dynamical variable. The intermolecular potential was obtained by taking a numerical average over 120 randomly chosen relative orientations of a pair of $\mathrm{C}_{60}$ molecules. The resulting potential was very similar to, but not the same as the Girifalco potential. The phase diagram obtained for this model was similar to the GEMC result of Hagen et $a l .,{ }^{3}$ i.e., the sublimation line passes $\sim 40 \mathrm{~K}$ above the liquid-vapor critical point in the $T-\rho$ plane and the liquid phase is thermodynamically unstable everywhere. However, this comparison should not be taken seriously because of the different models employed.

In addition to these simulation studies, theoretical predictions of the phase diagram of $\mathrm{C}_{60}$ have also been made using the integral equation method, ${ }^{9}$ the DFT of freezing, ${ }^{6,10}$ the thermodynamic perturbation theory ${ }^{11}$ and these 
combination. ${ }^{12}$ The integral equation method provides us with a powerful means of calculating the EOS of the fluid phase and is useful in the study of the liquid-vapor phase transition. ${ }^{13}$ of the various versions the Zerah-Hansen (often referred to as HMSA) and the modified hypernetted-chain (MHNC) theories have been used for the fluid $\mathrm{C}_{60}$ and yielded $T_{c} \approx 2050 \mathrm{~K}$ (Ref. 4) and $T_{c} \approx 1920 \mathrm{~K},{ }^{9}$ respectively, for the critical temperature, the latter being in better agreement with the simulations. ${ }^{4,7}$ More extensive study has been made by Hasegawa and Ohno $^{10}$ using the generalized MWDA (GMWDA), a version of the DFT of freezing. ${ }^{14}$ The GMWDA is an extension of the MWDA (Ref. 15) (modified weighted-density-approximation) and works for most systems. ${ }^{16}$ In this theory the EOS of a uniform fluid was calculated using the MHNC theory and the result for the liquid-vapor coexistence was similar to that of Caccamo 9 obtained by essentially the same method. The triple point predicted by the GMWDA falls slightly $(\sim 20 \mathrm{~K})$ below the critical point and the liquid phase was found to exist in qualitative agreement with the MD result of Cheng et $a l^{4}$

The work of Mederos and Navascués ${ }^{12}$ was based on the SPWDA, a simplified version of the perturbation weighteddensity approximation (PWDA), and yielded $T_{c} \approx 2200 \mathrm{~K}$ and $T_{t} \approx 1930 \mathrm{~K}$. For a uniform fluid this theory reduces to a thermodynamic perturbation theory. Such a theory works quite well for the Lennard-Jones (LJ) fluid, ${ }^{17,18}$ but yields too high critical temperature for $\mathrm{C}_{60}$ compared with the results of more accurate integral equation methods. ${ }^{11}$

Finally, Tau et al. ${ }^{19}$ took a somewhat different approach to explore the phase diagram of $\mathrm{C}_{60}$. They used the so-called hierarchical reference theory (HRT) which has been devised to deal with long-wavelength fluctuations using a basic concept of the renormalization group ( $R G$ ) theory. At present, the RG theory is only the way to properly treat unusual fluid behavior near the critical point. ${ }^{20}$ The HRT result for $T_{c}$ was $2138 \mathrm{~K}$, which is higher than the HMSA and the MHNC results by $\sim 90 \mathrm{~K}$ and $\sim 200 \mathrm{~K}$, respectively. This result of high $T_{c}$ is possibly due to the perturbative treatment of the long-range part of the intermolecular potential in the HRT as actually it is in the thermodynamic perturbation theories. $^{11,12,18}$

The results for $\mathrm{C}_{60}$ have led to more systematic investigations on the relation between the nature of intermolecular potential and the phase behavior. These investigations are twofold; the first is concerned with the disappearance of stable liquid phase, ${ }^{21,22}$ and the second with the isostructural solid-solid transition which occurs in systems with an extremely short-ranged potential. ${ }^{18,23-25}$ The previous investigations on these subjects are summarized in Ref. 18.

In the present work we performed extensive $\mathrm{MC}$ simulations for both fluid and solid phases of $\mathrm{C}_{60}$ and made a full free-energy analysis to explore the high-temperature phase diagram. As we have discussed above the previous simulations have yielded different results for the phase diagram of $\mathrm{C}_{60}$, which is in contrast to the case of the $\mathrm{LJ}$ system for which the most extensive simulation studies have been made. This difference may be explained by the nature of the intermolecular potential of $\mathrm{C}_{60}$, i.e., the strong repulsion at short range and the relatively short-ranged attractive part. Simula- tions for such a system are generally less efficient in the sense that the equilibration time is relatively long and a large number of steps are required in order to achieve numerical stability of any physical quantity of interest. We find that the strong repulsion at short range prevents us to make an accurate computation of the virial pressure and is the major source of uncertainty in simulations. In the case of constantpressure simulations, either MC or MD, the uncertainty of the virial pressure inevitably leads to that of the volume. This difficulty can partly be avoided by taking the energy route in which the excess (Helmholtz) free energy is obtained by performing isochoric integration of the internal energy. The excess internal energy of the fluid $\mathrm{C}_{60}$ obtained in the MC simulations is numerically much more stable than the virial pressure and the resulting free energy is also less uncertain than that obtained by integrating the virial pressure in the pressure route. The energy route is also useful to calculate the EOS on the high-density side of the liquid-vapor coexistence region. The MC result for the virial pressure is relatively stable for the solid phase and the pressure route may safely be used.

In the next section we present the outline of the MC method employed in the present study. We then present, in Sec. III, the results of our simulations and the methods of analyses used to obtain the phase diagram. The final section is devoted to the summary and concluding remarks.

\section{OUTLINE OF THE MC SIMULATIONS}

We used the Girifalco potential ${ }^{5}$ as the intermolecular potential of $\mathrm{C}_{60}$. This potential has been obtained by assuming free rotation of rigid $\mathrm{C}_{60}$ and the $\mathrm{LJ}$ potential as the interaction for a pair of carbon atoms on different $\mathrm{C}_{60} \mathrm{~mol}-$ ecules. The result is given by

$$
\begin{aligned}
\phi(r)= & -A\left[\frac{1}{s(s-1)^{3}}+\frac{1}{s(s+1)^{3}}-\frac{2}{s^{4}}\right] \\
& +B\left[\frac{1}{s(s-1)^{9}}+\frac{1}{s(s+1)^{9}}-\frac{2}{s^{10}}\right],
\end{aligned}
$$

where $s=r / \sigma, \sigma$ being the diameter of the $\mathrm{C}_{60}$ molecule $(\sigma=0.71 \mathrm{~nm})$. The parameters $A$ and $B$ in Eq. (1) have been determined empirically ${ }^{5}$ and given by $A=74.94 \times 10^{-15} \mathrm{erg}$ and $B=135.95 \times 10^{-18} \mathrm{erg}$. The Girifalco potential is useful for the orientationally disordered phase at high temperature and, as we have discussed in the previous section, has been used in most simulations and theoretical calculations for the high-temperature phase diagram of $\mathrm{C}_{60}$. The constant-NVT ensemble $\mathrm{MC}$ method was employed in the present work and, unless otherwise stated, the simulations were performed using the particle number of $N=256$ in the cubic cell of volume $V$ subject to the periodic boundary condition.

\section{A. Fluid phase}

We took the energy route for calculating the free energy of fluid. In this method the excess free energy $F_{\text {ex }}$ is calculated as

$$
\frac{F_{\mathrm{ex}}(\rho, T)}{N k_{B} T}=\frac{F_{\mathrm{ex}}\left(\rho, T_{0}\right)}{N k_{B} T_{0}}-\int_{T_{0}}^{T} \frac{U_{\mathrm{ex}}\left(\rho, T^{\prime}\right)}{N k_{B} T^{\prime}} \frac{d T^{\prime}}{T^{\prime}},
$$


where $\rho=N / V$, the particle number density, and $U_{\mathrm{ex}}$ is the excess internal energy, i.e., the thermal average of the interaction energy,

$$
U_{\mathrm{ex}}(\rho, T)=\left\langle\sum_{i<j} \phi\left(r_{i j}\right)\right\rangle .
$$

In Eq. (2) $T_{0}$ is taken to be large enough that the corresponding free energy can easily be calculated or obtained exactly. Here we used the limit $T_{0} \rightarrow \infty$ as we discuss below.

Another way of calculating the free energy is to take the pressure route, in which $F_{\mathrm{ex}}$ is calculated as

$$
\frac{F_{\mathrm{ex}}(\rho, T)}{N k_{B} T}=\frac{F_{\mathrm{ex}}\left(\rho_{0}, T\right)}{N k_{B} T}+\int_{\rho_{0}}^{\rho}\left[\frac{p\left(\rho^{\prime}, T\right)}{\rho^{\prime} k_{B} T}-1\right] \frac{d \rho^{\prime}}{\rho^{\prime}},
$$

where $p$ is the virial pressure directly obtained in simulation,

$$
p(\rho, T)=\rho k_{B} T\left\{1-\frac{1}{3 N}\left\langle\sum_{i<j} r_{i j} \frac{\partial \beta \phi\left(r_{i j}\right)}{\partial r_{i j}}\right\rangle\right\},
$$

with $\beta=1 / k_{B} T$. We have $F_{\text {ex }}\left(\rho_{0}, T\right)=0$ in the limit $\rho_{0} \rightarrow 0$ and this limit can be used to calculate $F_{\text {ex }}(\rho, T)$ above the liquid-vapor critical temperature, $T_{c}$, and on the lowdensity side of the two-phase region. The integration in Eq. (4) cannot be performed across the two-phase region, at least in principle, and the use of the energy route must be supplemented to calculate $F_{\text {ex }}(\rho, T)$ on the high-density side.

The energy route was consistently used to calculate the EOS of the fluid phase, although the pressure route was also used for comparisons and for testing the internal consistency where possible. In the limit, $T_{0} \rightarrow \infty, F_{\text {ex }}\left(\rho, T_{0}\right)$ does not vanish in the case of $\mathrm{C}_{60}$ but approaches the hard-sphere (HS) result since $\phi(r)$ diverges at $r=\sigma$. We may use the Carnahan-Starling ${ }^{26}$ (CS) EOS for the HS system,

$$
\frac{F_{\mathrm{ex}}(\rho, T)}{N k_{B} T}=\frac{\eta(1-3 \eta)}{(1-\eta)^{2}}
$$

where $\eta$ is the packing fraction defined by $\eta=(\pi / 6) \rho \sigma^{3}$. At $\rho=1 \mathrm{~nm}^{-3}$, nearly the highest fluid density of interest, we have $\eta=0.1874$. The above $C S$ result is virtually exact at this and smaller values of $\eta$. Then, Eq. (2) is conveniently written as

$$
\beta f_{\mathrm{ex}}(\rho, T)=\frac{\eta(1-3 \eta)}{(1-\eta)^{2}}+\int_{0}^{\beta} \beta^{\prime} u_{\mathrm{ex}}\left(\rho, T^{\prime}\right) \frac{d \beta^{\prime}}{\beta^{\prime}}
$$

where $f_{\mathrm{ex}}=F_{\mathrm{ex}} / N$ and $u_{\mathrm{ex}}=U_{\mathrm{ex}} / N$.

The integral in Eq. (7) cannot directly be performed using simulation data of $u_{\mathrm{ex}}$ since the integrand is singular in the limit $\beta^{\prime} \rightarrow 0$. We can easily see this singularity using the expression for $u_{\mathrm{ex}}$,

$$
\beta^{\prime} u_{\mathrm{ex}}\left(\rho, T^{\prime}\right)=2 \pi \rho \int_{0}^{\infty} r^{2} \beta^{\prime} \phi(r) g\left(r ; \rho, T^{\prime}\right) d r,
$$

where $g(r)$ is the radial distribution function. In the limit, $\beta^{\prime} \rightarrow 0, g(r)$ may be approximated as 27

$$
g\left(r ; \rho, T^{\prime}\right)=g_{\mathrm{HS}}(r / \sigma ; \eta) \exp \left[-\beta^{\prime} \phi(r)\right],
$$

$g_{\text {HS }}$ being the HS radial distribution function. Noting that, for the Girifalco model in Eq. $(1), \phi(r) \approx B / s(s-1)^{9}$ in the vicinity of $s=r / \sigma=1$ and that this behavior of $\phi(r)$ yields the leading contribution to the integral of Eq. (8) in the limit $\beta^{\prime} \rightarrow 0$, we have

$$
\begin{aligned}
\beta^{\prime} u_{\mathrm{ex}}\left(\rho, \beta^{\prime}\right) \rightarrow & 2 \pi \rho \sigma^{3} g_{\mathrm{HS}}(1 ; \eta) \\
& \times \int_{1}^{\infty} \frac{\beta^{\prime} B}{(s-1)^{9}} \exp \left[-\frac{\beta^{\prime} B}{(s-1)^{9}}\right] d s \\
= & 12 \eta g_{\mathrm{HS}}(1 ; \eta) \frac{\left(B \beta^{\prime}\right)^{1 / 9}}{9} I_{9},
\end{aligned}
$$

where, according to the CS result, ${ }^{26}$

$$
g_{H S}(1 ; \eta)=\frac{1-\eta / 2}{(1-\eta)^{3}}
$$

and

$$
I_{n}=\int_{0}^{\infty} x^{-1 / n} e^{-x} d x
$$

with $I_{9}=1.07777$. We note that Eq. (10) is exact in the limit $\beta^{\prime} \rightarrow 0$ and the integrand in Eq. (7) diverges as $\beta^{\prime-8 / 9}$.

In Eq. (7) the integration over the range of small $\beta^{\prime}$ can conveniently be performed using a new dimensionless variable, $y=\left(\beta^{\prime} / \beta_{R}\right)^{1 / 9}=\left(T_{R} / T^{\prime}\right)^{1 / 9}, T_{R}$ being the reference temperature introduced for convenience and taken to be $T_{R}$ $=1000 \mathrm{~K}$, i.e.,

$$
\begin{aligned}
\beta \Delta f_{\mathrm{ex}}(\rho, T) & \equiv \int_{0}^{\Delta \beta} \beta^{\prime} u_{\mathrm{ex}}\left(\rho, T^{\prime}\right) \frac{d \beta^{\prime}}{\beta^{\prime}} \\
& =9 \int_{0}^{\Delta y} \beta^{\prime} u_{\mathrm{ex}}\left(\rho, T^{\prime}\right) \frac{d y}{y},
\end{aligned}
$$

where $\Delta y=\left(\Delta \beta / \beta_{R}\right)^{1 / 9}$ and, for brevity, $\beta^{\prime}\left(=\beta_{R} y^{9}\right)$ is retained in the integrand. Using Eq. (10) we have, in the limit $\beta^{\prime} \rightarrow 0(y \rightarrow 0)$,

$$
\frac{\beta^{\prime} u_{\mathrm{ex}}\left(\rho, T^{\prime}\right)}{y} \rightarrow \frac{4}{3} \eta g_{\mathrm{HS}}(1 ; \eta)\left(\beta_{R} B\right)^{1 / 9} I_{9},
$$

and this result can be used to perform the integration in Eq. (13). As we actually show later, the MC data for $\beta^{\prime} u_{\mathrm{ex}} / y$ at finite $y$ extrapolate quite well to the limiting value given by Eq. (14). The integration in Eq. (7) over the remaining interval, $\Delta \beta<\beta^{\prime}<\beta$, can directly be performed using simulation data for $u_{\mathrm{ex}}$.

\section{B. Solid phase}

For the solid phase we took the pressure route and calculated the free energy using Eqs.(4) and (5). The simulation results for the virial pressure are relatively stable for the solid phase and the thermodynamic integration in Eq. (4) is limited to a narrow range of the density if we choose an appropriate density $\rho_{0}$ within the solid phase. The absolute free energy at $\rho_{0}$ was calculated using a coupling-constant method in which we used the classical Einstein solid consisting of the particles with the same mass as that of $\mathrm{C}_{60} .^{28}$ Using a linear path connecting the $\mathrm{C}_{60}$ and Einstein solids whose potential energies are denoted as $U$ and $U_{E}$, respectively, we have 


$$
F\left(\rho_{0}, T\right)=F_{E}\left(\rho_{0}, T\right)+\int_{0}^{1} d \lambda\left\langle U-U_{E}\right\rangle_{\lambda},
$$

where $F_{E}$ is the free energy of the Einstein solid and $\langle\cdots\rangle_{\lambda}$ represents the thermal average in the system with the Hamiltonian,

$$
H_{\lambda}=H_{E}+\lambda\left(U-U_{E}\right),
$$

$H_{E}$ being the Hamiltonian of the Einstein solid. The free energy of the Einstein solid per particle is given by ${ }^{28}$

$$
\begin{aligned}
\beta f_{E}\left(\rho_{0}, T\right)= & \beta u_{0}\left(\rho_{0}\right)+\frac{3}{2} \ln \left[\frac{3 \Lambda^{2}}{2 \pi\left\langle(\Delta r)^{2}\right\rangle_{E}}\right] \\
= & \beta u_{0}\left(\rho_{0}\right)+\beta f_{\mathrm{id}}\left(\rho_{0}, T\right)+1 \\
& +\frac{3}{2} \ln \left[\frac{3}{2 \pi \rho_{0}^{2 / 3}\left\langle(\Delta r)^{2}\right\rangle_{E}}\right]
\end{aligned}
$$

where $u_{0}$ is the potential energy per particle of the static crystal, $\Lambda$ is the thermal de Broglie wavelength, $\left\langle(\Delta r)^{2}\right\rangle_{E}$ $=\left\langle(\Delta r)^{2}\right\rangle_{\lambda=0}$ is the mean-square-displacement (MSD) of the pure Einstein solid, and $f_{\text {id }}$ is the ideal-gas free energy, $\beta f_{\text {id }}\left(\rho_{0}, T\right)=\ln \left(\rho_{0} \Lambda^{3}\right)-1$. The MSD, $\left\langle(\Delta r)^{2}\right\rangle_{E}$, characterizes the Einstein model and its optimal choice may be ${ }^{28}$

$$
\left\langle(\Delta r)^{2}\right\rangle_{E}=\left\langle(\Delta r)^{2}\right\rangle,
$$

where $\left\langle(\Delta r)^{2}\right\rangle=\left\langle(\Delta r)^{2}\right\rangle_{\lambda=1}$ is the MSD of the true system and obtained by simulations.

The term $\langle U\rangle_{\lambda}$ in the integrand of Eq. (15) diverges in the limit $\lambda \rightarrow 0$ and the integration cannot be performed using numerical data. However, by an appropriate choice of $\left\langle(\Delta r)^{2}\right\rangle_{E}$ such as in Eq. (18), the singularity can be eliminated for an ordinary potential diverging at zero separation. ${ }^{28}$ It is not immediately obvious if it is also the case for the Girifalco potential which diverges at $r=\sigma$, and it may be useful to explicitly show that the singularity can also be eliminated for $\mathrm{C}_{60}$. To this end we note that $u_{\lambda}=\langle U\rangle_{\lambda} / N$ can be written as

$$
\beta u_{\lambda}=\frac{1}{2 N} \int d \mathbf{r}_{1} \int d \mathbf{r}_{2} \beta \phi\left(r_{12}\right) \rho_{\lambda}\left(\mathbf{r}_{1}\right) \rho_{\lambda}\left(\mathbf{r}_{2}\right) g_{\lambda}\left(\mathbf{r}_{1}, \mathbf{r}_{2}\right),
$$

where $\rho_{\lambda}$ and $g_{\lambda}$ are, respectively, the density distribution and the pair correlation function of the system with the Hamiltonian given by Eq. (16). In the limit $\lambda \rightarrow 0, \rho_{\lambda}(\mathbf{r})$ is nothing but the density distribution in the Einstein solid and given by the superposition of the Gaussians centered on lattice sites,

$$
\rho_{\lambda}(\mathbf{r})=\left(\frac{\alpha}{\pi}\right)^{3 / 2} \sum_{i} \exp \left[-\alpha\left(\mathbf{r}-\mathbf{R}_{i}\right)^{2}\right]
$$

where $\mathbf{R}_{i}$ is the $i$ th lattice site of a given crystal structure and $\alpha^{-1}=3\left\langle(\Delta r)^{2}\right\rangle_{E} / 2,\left\langle(\Delta r)^{2}\right\rangle_{E}$ being given by Eq. (18) in the present choice. For the purpose of estimating an order of magnitude of $\langle U\rangle_{\lambda}$ we may use an approximation,

$$
g_{\lambda}\left(\mathbf{r}_{1}, \mathbf{r}_{2}\right) \approx \exp \left[-\lambda \beta \phi\left(r_{12}\right)\right],
$$

which reduces to $g_{\lambda}\left(\mathbf{r}_{1}, \mathbf{r}_{2}\right)=1$ in the limit $\lambda \rightarrow 0$, in agreement with the result for the Einstein solid. Using Eqs. (20) and (21) into Eq. (19) we have $e^{29}$

$$
\begin{aligned}
\beta u_{\lambda} \approx & \frac{1}{2}\left(\frac{\alpha}{2 \pi}\right)^{1 / 2} \sum_{R_{j} \neq 0} \frac{N_{j}}{R_{j}} \int_{0}^{\infty} d r r \beta \phi(r) \exp [-\lambda \beta \phi(r)] \\
& \times\left\{\exp \left[-\frac{\alpha}{2}\left(r-R_{j}\right)^{2}\right]-\exp \left[-\frac{\alpha}{2}\left(r+R_{j}\right)^{2}\right]\right\} \\
\approx & \frac{1}{2}\left(\frac{\alpha}{2 \pi}\right)^{1 / 2} \frac{N_{1}}{R_{1}} \int_{0}^{\infty} d r r \beta \phi(r) \exp [-\lambda \beta \phi(r)] \\
& \times \exp \left[-\frac{\alpha}{2}\left(r-R_{1}\right)^{2}\right],
\end{aligned}
$$

where $R_{j}$ and $N_{j}$ are, respectively, the distance and the number of the $j$ th nearest-neighbor lattice sites, and only the leading term is retained in the second step. The omitted terms in Eq. (22) are extremely small since $\alpha$ is very large as actually it is. Using an analysis similar to that leading to Eq. (10), we have

$\beta u_{\lambda} \rightarrow \frac{1}{2}\left(\frac{\alpha^{*}}{2 \pi}\right)^{1 / 2} \frac{N_{1}}{R_{1}^{*}} \exp \left[-\frac{\alpha^{*}}{2}\left(1-R_{1}^{*}\right)^{2}\right] \frac{(\beta B)^{1 / 9}}{9 \lambda^{8 / 9}} I_{9}$,

showing that $u_{\lambda}$ strongly diverges as $\lambda^{-8 / 9}$. In Eq. (23), $\alpha^{*}=\alpha \sigma^{2}, R_{1}^{*}=R_{1} / \sigma$, and $I_{9}$ is defined by Eq. (12). The above result is somewhat different from that for a potential which diverges at zero separation. For a typical example of the LJ potential, $\phi(r)=4 \epsilon\left[(\sigma / r)^{12}-(\sigma / r)^{6}\right]$, we have

$$
\beta u_{\lambda} \rightarrow 2 \alpha^{*}\left(\frac{\alpha^{*}}{2 \pi}\right)^{1 / 2} N_{1} \exp \left(-\frac{1}{2} \alpha^{*} R_{1}^{* 2}\right) \frac{(4 \beta \epsilon)^{1 / 4}}{12 \lambda^{3 / 4}} I_{4},
$$

where $I_{4}$ is again defined by Eq. (12), and $\alpha^{*}$ and $R_{1}^{*}$ are now defined in terms of the LJ potential parameter, $\sigma$. With a proper choice of $\left\langle(\Delta r)^{2}\right\rangle_{E}$ as in Eq. (18) the singular behavior of $u_{\lambda}$ in Eq. (24) contributes very little to the integral in Eq. (15) owing to the vanishingly small prefactor, $\exp \left(-\alpha^{*} R_{1}^{2} / 2\right)$, in other words, small overlap of the densities associated with the neighboring lattice sites. ${ }^{28}$ The present case of Eq. (23) is apparently different, but the prefactor $\exp \left[-\alpha^{*}\left(1-R_{1}^{*}\right)^{2} / 2\right]$ is also vanishingly small as we actually demonstrate in the next section and the singular behavior of $u_{\lambda}$ in the vicinity of $\lambda=0$ can be ignored.

\section{RESULTS AND DISCUSSION}

Figure 1 shows the MC simulation results for the integrand in Eq. (13). The upper bound of the integral, $\Delta y$ $=(0.01)^{1 / 9}=0.5995$, corresponds to $T=1 \times 10^{5} \mathrm{~K}$ with the choice $T_{R}=1000 \mathrm{~K}$, about 50 times higher than the temperatures of interest for $\mathrm{C}_{60}$. The values of $\beta u_{\mathrm{ex}} / y$ at finite $y$ extrapolate quite well to the limiting value given by Eq. (14), confirming the consistency between the theoretical analysis and the simulations. The HS term in Eq. (7) and the hightemperature contribution given by Eq. (13) are the same order of magnitude, and together account for the substantial part of the excess free energy at temperatures of interest $(\sim 2000 \mathrm{~K})$. Figure 2 illustrates the simulation results for the 

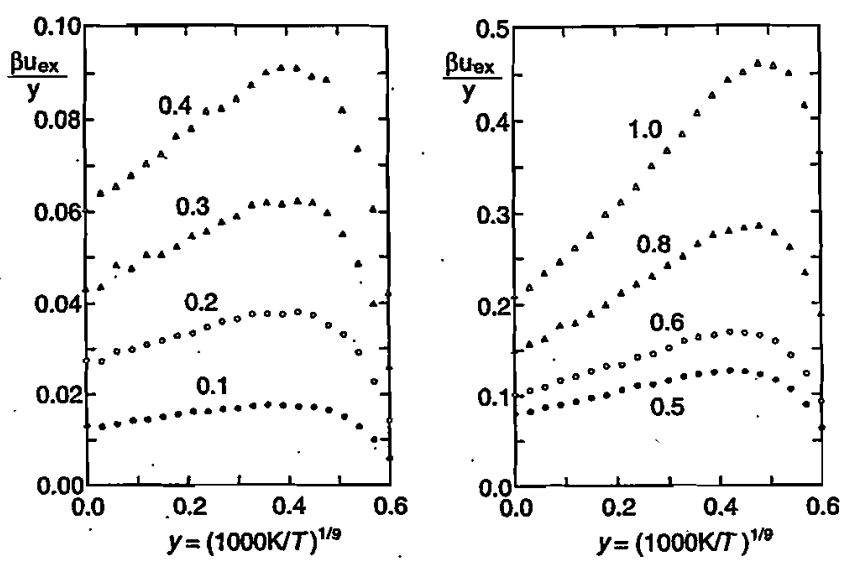

FIG. 1. MC simulation results for the integrand in Eq. (13). The values at $y=0$ were obtained from Eq. (14) and the parameter labeling each curve represents the density in unit of $\mathrm{nm}^{-3}$. The upper bound of the integral in Eq. (13), $\Delta y=(0.01)^{1 / 9}=0.5995$, corresponds to $T=1 \times 10^{5} \mathrm{~K}$.

excess internal energy in the low-temperature range, $T<1$ $\times 10^{5} \mathrm{~K}$. At a temperature below $\sim 2000 \mathrm{~K}$ depending on the density, $\beta u_{\mathrm{ex}}$ begins to show a large fluctuation or a drastic change, suggesting that the system becomes unstable against the phase separation. We should note that we can locate neither the liquid-vapor nor the solid-vapor phase boundary using such a instability observed in constant-volume simulations for a finite number of particles. In such a simulation the system remains uniform and stable until it reaches a point deep inside the true coexistence region since the formation of interface is energitically unfavorable. Abramo and Coppolino ${ }^{30}$ disregarded this feature and were completely wrong in the interpretation of their MD simulation results for $\mathrm{C}_{60}$.

Figure 3 shows the typical examples of the excess free energy obtained from Eqs. (7) and (13) using the simulation data for $\beta u_{\mathrm{ex}}$ as shown in Figs. 1 and 2. In the present analysis to obtain the phase diagram these MC results of the excess free energy were fitted to a polynomial,

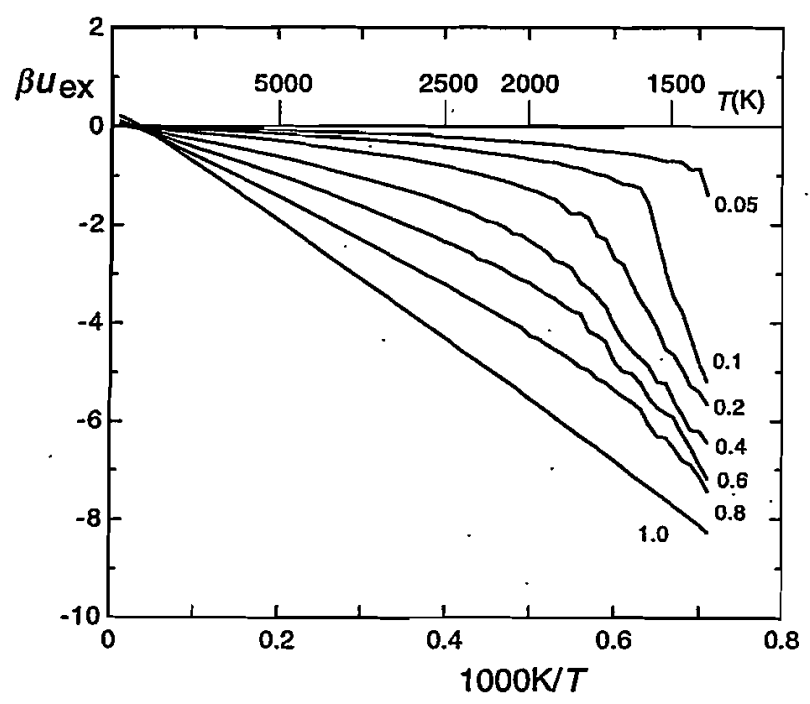

FIG. 2. MC simulation results for the excess internal energy. The parameter labelling each curve represents the density in unit of $\mathrm{nm}^{-3}$.

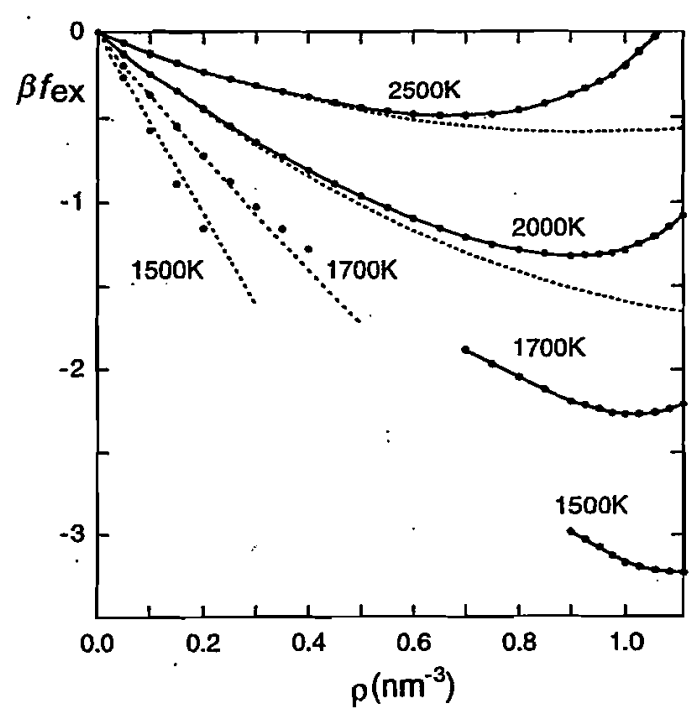

FIG. 3. The isotherms of the excess free energy obtained from the present MC simulations through Eq. (7) (filled circles) and from the low-order virial expansion (dotted curves), $\beta f_{\text {ex }}(\rho, T)=A_{2} \rho+A_{3} \rho^{2}$, with the exact $A_{2}=B_{2}$ and $A_{3}=B_{3} / 2$. The full curves are the least-mean-square fit to the MC results (see the text).

$$
\beta f_{\text {ex }}(\rho, T)=A_{2} \rho+A_{3} \rho^{2}+A_{4} \rho^{3}+\cdots+A_{m} \rho^{m-1} .
$$

The corresponding pressure is given by

$$
\begin{aligned}
p & =\rho k_{B} T\left(1+\rho \frac{\partial \beta f_{\mathrm{ex}}}{\partial \rho}\right) \\
& =\rho k_{B} T\left(1+B_{2} \rho+B_{3} \rho^{2}+B_{4} \rho^{3}+\cdots+B_{m} \rho^{m-1}\right),
\end{aligned}
$$

where $B_{n}=(n-1) A_{n}$. In this fitting procedure we used the exact virial coefficients for $B_{2}=A_{2}$ and $B_{3}=2 A_{3}$, which can easily be calculated as $^{27}$

$$
B_{2}(T)=-2 \pi \int_{0}^{\infty} r^{2} f(r) d r
$$

and

$$
\begin{aligned}
B_{3}(T)= & -\frac{8 \pi^{2}}{3} \int_{0}^{\infty} d r_{1} r_{1} f\left(r_{1}\right) \int_{0}^{\infty} d r_{2} r_{2} f\left(r_{2}\right) \\
& \times \int_{\left|r_{1}-r_{2}\right|}^{\left|r_{1}+r_{2}\right|} d r_{3} r_{3} f\left(r_{3}\right),
\end{aligned}
$$

where $f(r)$ is the Mayer function defined by $f(r)$ $=\exp [-\beta \phi(r)]-1$. The remaining coefficients, $A_{4}-A_{m}$, in Eq. (24a) were determined by the least-mean-square fit and we found that $m=7$ yields a very accurate fitting. The use of the exact $B_{2}$ and $B_{3}$ eliminates the arbitrariness of the fitting procedure and the resulting low-order coefficients, possibly $B_{4}$ and $B_{5}$, may be interpreted as the approximate virial coefficients. We note, however, that the coefficients $B_{n}$ with $n>3$ of the truncated expansion inevitably contain more or less the effect of high-order terms in the virial expansion. In Fig. 3 we find that the simulation results of $\beta f_{\text {ex }}$ at low densities are in good agreement with those obtained from the low-order virial expansion, $\beta f_{\mathrm{ex}}(\rho, T)=A_{2} \rho+A_{3} \rho^{2}$, with $A_{2}=B_{2}$ and $A_{3}=B_{3} / 2$, suggesting the accuracy of the present simulation results. 

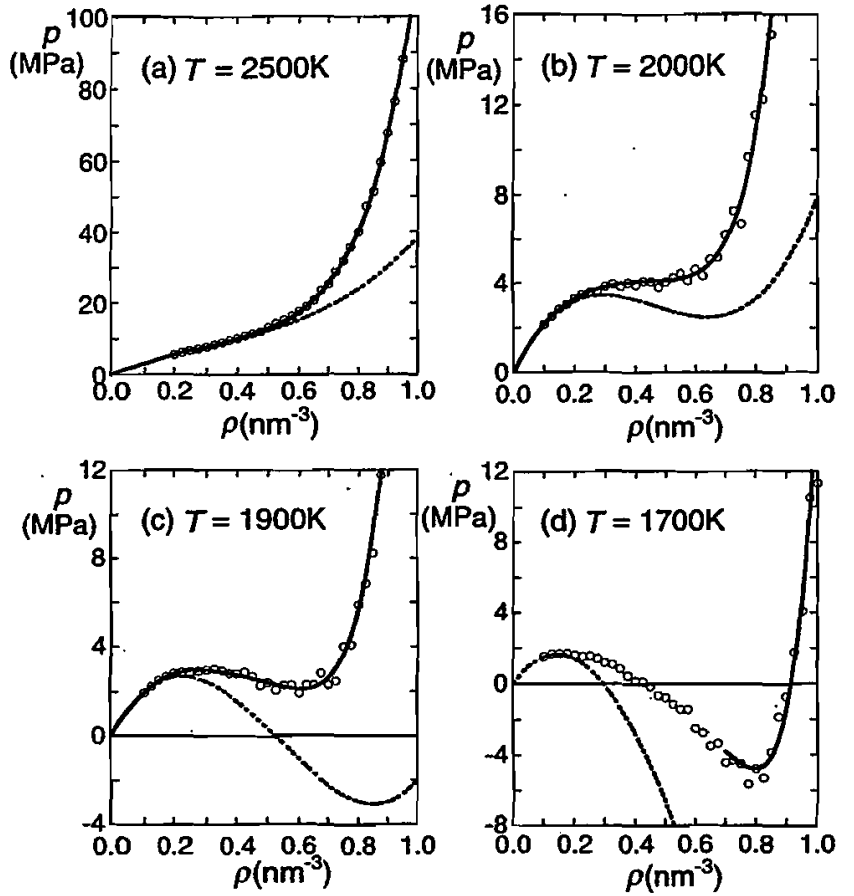

FIG. 4. Comparisons of the pressure from the present MC simulations through Eq. (25) (full curves), the virial pressure directly obtained in the MC simulations through Eq. (5) (open circles), and the low-order virial expansion, $p=\rho k_{B} T\left(1+B_{2} \rho+B_{3} \rho^{2}\right.$ ), with the exact $B_{2}$ and $B_{3}$ (dotted curves).

Figure 4 illustrates the typical examples of the pressure obtained from Eq. (25) and their comparisons with the virial pressure directly obtained in the simulations through Eq. (5). These results of the two routes are generally in agreement with each other at high temperatures. However, as temperature decreases the fluctuation of the virial pressure becomes large, which makes it difficult to obtain accurate EOS. We note that the simulation results for the virial pressure in Fig. 4 were obtained using an ensemble of $3 \times 10^{4} \mathrm{MC}$ steps $\left(7.68 \times 10^{6}\right.$ steps), whereas $1 \times 10^{4} \mathrm{MC}$ steps were sufficient to yield accurate excess internal energy in the energy route.

Next, we proceed to the simulation results for the solid phase. Following the previous simulations ${ }^{3,4}$ and theoretical calculations, ${ }^{6,10,12}$ we assumed a face-centered-cubic (fcc) structure. The recent $\mathrm{MD}$ simulations have shown that the model $\mathrm{C}_{60}$ at high temperatures actually freezes into a fcc structure as the density increases, ${ }^{30}$ in agreement with the present assumption. The absolute free energies of the solid were calculated using the prescriptions given in the previous section. Figures 5 and 6 illustrate the process of such calculations. For a typical solid $\mathrm{C}_{60}$ at $T=2000 \mathrm{~K}$ and $\rho=1.25$ $\mathrm{nm}^{-3}$, we have $\alpha^{*}=\alpha \sigma^{2} \approx 133$ from the simulations and hence $\exp \left[-\alpha^{*}\left(1-R_{1}^{*}\right)^{2} / 2\right] \approx 4.85 \times 10^{-7}$, which implies that the singular behavior of $\langle U\rangle_{\lambda}$ in the vicinity of $\lambda=0$ can safely be ignored in performing the integration in Eq. (15) and we may assume a smooth function for $\langle U\rangle_{\lambda}$. We actually fitted $\langle U\rangle_{\lambda}$ to a third-order polynomial of $\lambda$ with the coefficients determined by the simulation data at $\lambda=0.025$, $0.05,0.75$, and 0.1 . The value of $\langle U\rangle_{\lambda=0}$ in Fig. 5 is the result obtained in this way. Figure 6 shows the typical ex-

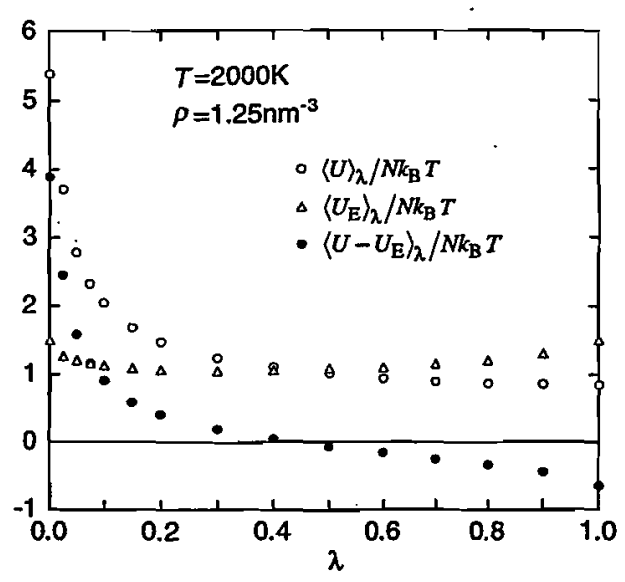

FIG. 5. MC simulation results for the integrand in Eq. (15). The value of $\langle U\rangle_{\lambda}$ at $\lambda=0$ was obtained using a third-order polynomial of $\lambda$ with the coefficients determined by the values at finite $\lambda$ (see the text for detail).

amples of the simulation data for the virial pressure. In contrast to the case of the fluid phase, the fluctuation of the data becomes less serious as temperature decreases, which seems to reffect that the solid is more stable at lower temperatures.

In the calculation of the phase diagram these results for the pressure of solid phase were fitted to a function of the form,

$$
p=\rho k_{B} T\left[1+b_{0}+b_{1}\left(\rho-\rho_{0}\right)+b_{2}\left(\rho-\rho_{0}\right)^{2}\right],
$$

using the least-mean-square method. The corresponding excess free energy is given by

$$
\begin{aligned}
\beta f_{\mathrm{ex}}(\rho, T)= & \beta f_{\mathrm{ex}}\left(\rho_{0}, T\right)+b_{0}\left(\rho-\rho_{0}\right)+\frac{1}{2} b_{1}\left(\rho-\rho_{0}\right)^{2} \\
& +\frac{1}{3} b_{2}\left(\rho-\rho_{0}\right)^{3}
\end{aligned}
$$

where $\beta f_{\text {ex }}\left(\rho_{0}, T\right)$ was calculated by the method given in the previous section and illustrated in Fig. 5.

Finally, Figs. 7 and 8 show the high-temperature phase diagram of $\mathrm{C}_{60}$ determined using all the results for the absolute free energy and the pressure of both phases. We first note that the resulting phase boundaries are quite systematic. It is difficult to estimate error bars in our calculations, but

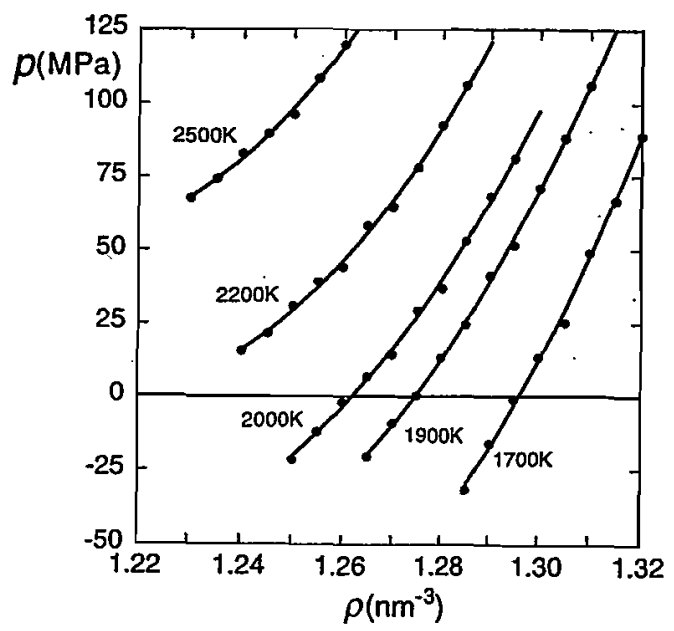

FIG. 6. MC simulation results for the virial pressure of the solid phase (filled circles) and the least-mean-square fit (full curves) to the MC data. 


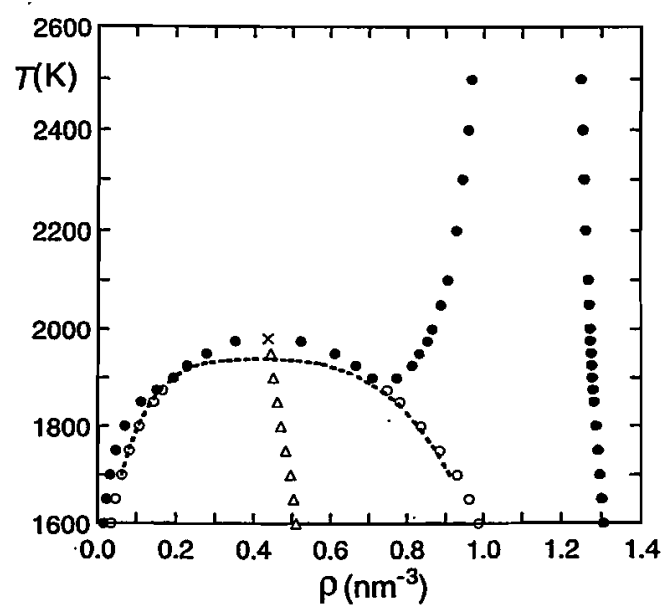

FIG. 7. High-temperature phase diagram of $\mathrm{C}_{60}$ in the $T-\rho$ plane obtained in the present MC simulations; the filled circles represent the stable phase boundary, the open circles the metastable liquid-vapor binodal points, the open triangles the mean density of the coexisting liquid and vapor, and the cross the estimated the liquid-vapor critical point. The dotted curve is the smooth interpolation of the GEMC (Ref. 7) results for $N=1500$.

they are possibly no larger than the size of circles used to locate the phase boundaries. The liquid-vapor critical point was determined by assuming a law,

$$
\Delta \rho \propto\left(T_{c}-T\right)^{\beta} \text {, }
$$

where $\Delta \rho=\rho_{1}-\rho_{v}$, the difference between the densities of the coexisting liquid and vapor, $T_{c}$ is the critical temperature, and $\beta$ the critical exponent (not the inverse temperature). We used the values of $\Delta \rho$ at $1900 \mathrm{~K}, 1925 \mathrm{~K}$, and $1950 \mathrm{~K}$ to determine $T_{c}$ and $\beta$ and found that $T_{c} \approx 1980 \mathrm{~K}$ and $\beta \approx 0.439$. In this determination we avoided the use of $\Delta \rho$ at the topmost temperature, $1975 \mathrm{~K}$, which is somewhat uncertain. Using the above results and the rectilinear law we obtained $\rho_{c} \approx 0.440 \mathrm{~nm}^{-3}$ for the critical density.

In Fig. 7 the present result for the liquid-vapor binodal line is compared with the GEMC result of Caccamo et al. ${ }^{7}$ obtained for $N=1500$, the largest system studied by them. We find that both are in good agreement with each other except in the critical region. In this comparison we should

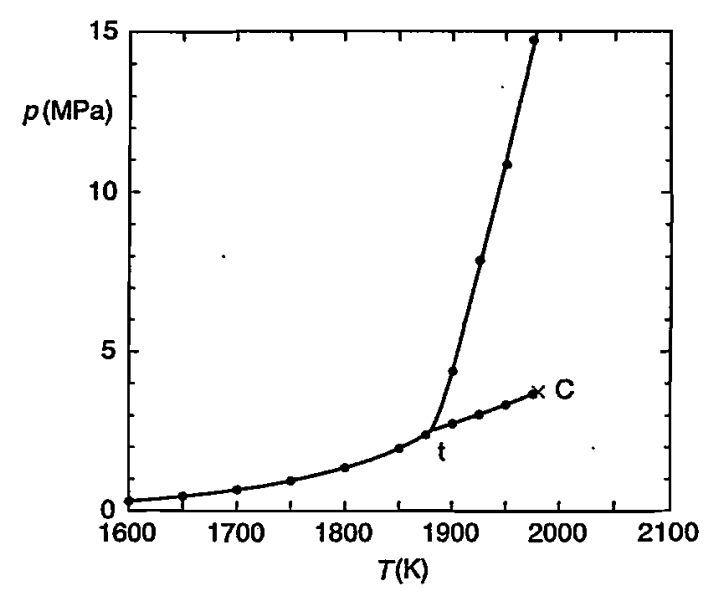

FIG. 8. High-temperature phase diagram of $\mathrm{C}_{60}$ in the $p-T$ plane; the filled circles represent the present MC simulation results, the full lines their smooth interpolation, and the cross the estimated liquid-vapor critical point. note that the GEMC results show some size-dependence and the agreement is worse for smaller $N$ and that the nonclassical exponent, ${ }^{19} \beta=0.32$, was used in the analysis of the GEMC data. The latter point will be discussed below in more detail. To see the $N$-dependence of the binodal line we also performed MC simulations for a larger system of $N=500$ and found that the result was almost indistinguishable from that for $N=256$ below $\sim 1900 \mathrm{~K}$. Above $1900 \mathrm{~K}$ we found small differences but they are almost invisible on the scale of Fig. 7; more explicitly, we obtained $T_{c}=1976 \mathrm{~K}, \beta=0.436$, and $\rho_{c}=0.445$ for $N=500$ using the data of $\Delta \rho$ at $1900 \mathrm{~K}$, $1925 \mathrm{~K}$, and $1950 \mathrm{~K}$ as in the case of $N=256$. These results suggest that $T_{c}$ has a tendency to lower with increasing $N$ in contrast to the GEMC results of Caccamo et al. ${ }^{7}$ However, the $N$-dependence predicted in our approach might be within the numerical uncertainty and could be marginal. To see the $N$-dependence more clearly we need to perform systematic simulations for larger $N$, which are computationally too heavy to perform within a reasonable computer time.

A more detailed inspection of the liquid-vapor coexistence shows that the present result for the critical exponent, $\beta \approx 0.44$, is large compared with the accepted nonclassical value, ${ }^{19} \beta=0.32$, and rather close to the classical value, $\beta=0.50$, which is responsible for the sharpness of the binodal line obtained in our simulations. The mean-field-like theories and most perturbation theories are known to give the classical exponent, ${ }^{19}$ which is the consequence of the improper treatment of the long-wavelength density fluctuation. Simulation studies also inevitably suffer from a similar limitation and, to avoid this difficulty, the critical point is usually located by using the nonclassical exponent, $\beta=0.32$, together with the data of $\Delta \rho$ at subcritical temperatures. Caccamo et $a l^{7}$ actually followed this method and located the critical point using their data of $\Delta \rho$ below $\sim 1900 \mathrm{~K}$, the highest temperature at which binodal points were available in their GEMC simulations. For comparisons, we also assumed $\beta=0.32$ and obtained $T_{c} \approx 1954 \mathrm{~K}$, which is very close to the GEMC results, $T_{c} \approx 1940 \mathrm{~K}$, for $N=1500$. In this estimation we used the MC data of $\Delta \rho$ at $1875 \mathrm{~K}$ and $1900 \mathrm{~K}$ with the expectation that these data are not much affected by the critical fluctuation and by a possible finite-size effect, which is also the largest in the critical region. The above comparison, however, should not be taken seriously since we have no criterion to choose the data of $\Delta \rho$.

The triple point was found at $T_{t}=1880 \mathrm{~K}$ and $\rho_{t}$ $=0.74 \mathrm{~nm}^{-3}$ from the present $\mathrm{MC}$ simulations. As we have discussed above, we may expect that at temperatures below $\sim 1900 \mathrm{~K}$ the present MC data for the fluid EOS are almost free form the critical fluctuation and the finite-size effect, suggesting that the resulting triple point is rather definite and a stable liquid phase certainly exists in the substantial range of temperature above $1880 \mathrm{~K}$.

Finally, the present results for the critical and triple point are compared in Table I with those of the previous simulations and theoretical calculations. These comparisons show that all the simulations and the MHNC results for $T_{c}$ and $\rho_{c}$ based on the same Girifalco potential are in the range 1900$2000 \mathrm{~K}$ and $0.39-0.56 \mathrm{~nm}^{-3}$, respectively. We note that the MHNC theory was also used to calculate the fluid EOS in the 
TABLE I. Parameters of the critical and triple point of $\mathrm{C}_{60}$ obtained in the present work and their comparisons with those of the previous simulations and theoretical calculations. Note that the GEMC (Ref. 3) and the MD (Ref. 8) results are based on the truncated potential and on a different model, respectively (see the text).

\begin{tabular}{|c|c|c|c|c|c|c|c|c|}
\hline & \multicolumn{2}{|c|}{ Present work } & \multirow{2}{*}{$\begin{array}{l}\text { GEMC } \\
\text { (Ref. 7) }\end{array}$} & \multirow{2}{*}{$\begin{array}{l}\text { GEMC } \\
\text { (Ref. 3) }\end{array}$} & \multirow{2}{*}{$\begin{array}{c}\mathrm{MD} \\
\text { (Ref. 4) }\end{array}$} & \multirow{2}{*}{$\begin{array}{c}\text { MD } \\
\text { (Ref. 8) }\end{array}$} & \multirow{2}{*}{$\begin{array}{l}\text { MHNC } \\
\text { (Ref. 9) }\end{array}$} & \multirow{2}{*}{$\begin{array}{l}\text { GMWDA } \\
\text { (Ref. 10) }\end{array}$} \\
\hline & $N=256$ & $N=500$ & & & & & & \\
\hline$T_{c}(\mathrm{~K})$ & $\begin{array}{c}1980 \\
1954^{\mathrm{a}}\end{array}$ & 1976 & $1924-194 \mathrm{i}^{\mathrm{b}}$ & 1798 & $1900 \pm 100$ & 1850 & 1910 & 1960 \\
\hline$\rho_{c}\left(\mathrm{~nm}^{-3}\right)$ & $\begin{array}{l}0.439 \\
0.444^{\mathrm{a}}\end{array}$ & 0.445 & $0.39-0.42^{\mathrm{b}}$ & 0.41 & $0.56 \pm 0.06$ & 0.51 & 0.43 & 0.41 \\
\hline$T_{t}(\mathrm{~K})$ & 1880 & & $1875^{\mathfrak{c}}$ & No & $\sim 1774$ & No & $\cdots$ & 1940 \\
\hline$\rho_{t}\left(\mathrm{~nm}^{-3}\right)$ & 0.74 & & $0.73^{c}$ & No & $\sim 0.944$ & No & $\cdots$ & 0.53 \\
\hline
\end{tabular}

${ }^{a}$ Obtained by assuming the nonclassical critical exponent, $\beta=0.32$, and using the data for the binodal points at $1900 \mathrm{~K}$ and $1875 \mathrm{~K}$.

'The results for $N=300-1500$ in this order.

Estimated from the intersection of the GEMC binodal line for $N=1500$ and the freezing line (after extrapolation) of the present work (see Fig. 7).

GMWDA of Ref. 10. As we have already pointed out, the results of Hagen $e t$ al. ${ }^{3}$ and Broughton et al. ${ }^{8}$ do not serve as the direct comparisons with others since the former used a truncated Girifalco potential, whereas the latter employed a similar but somewhat different model.

\section{SUMMARY AND CONCLUSIONS}

We have presented the results of extensive MC simulations for the high-temperature phase diagram of the model $\mathrm{C}_{60}$. The present study has been motivated by the previous simulations for $\mathrm{C}_{60}$ performed in an attempt to give an answer to the question of whether this substance has a stable liquid phase or not. These simulations were generally subject to large uncertainties and yielded quantitatively or even qualitatively different results depending on the techniques employed or the treatments of the model, and the above answer has been inconclusive. In the present study, to avoid the difficulty inherent in these simulations, we consistently took the energy route to calculate the absolute free energy of the fluid phase. This route is computationally more demanding but enables us to make accurate calculations of the absolute free energy of the fluid phase at any relevant point in the $T-\rho$ plane. This method actually yielded numerically more stable results than that utilizing the virial pressure. For the solid $\mathrm{C}_{60}$ the above difficulty is less serious and we took the pressure route in which the free energy is obtained by integrating the virial pressure. We have also presented the detailed accounts of resolving some practical difficulties in the calculation of the free energy using simulation data. The general accounts of these practices might have been implicit in the previous simulation studies for the phase diagram ${ }^{28}$ but not ready for an immediate application to a particular case of $\mathrm{C}_{60}$.

The resulting phase diagram is in fact quite systematic and free from large uncertainty. The most difficult problem in determining the phase diagram is how to treat the effect of large density fluctuation in the liquid-vapor critical region. The constant $-N V T$ ensemble simulations are certainly not adequate for exploring this problem and seem to yield a classical result for the critical exponent, which is responsible for the sharpness of the binodal line obtained in the present work. We have bypassed this problem by using the data at subcritical temperatures and assuming the nonclassical critical exponent. The critical temperature estimated in this way was certainly above $1950 \mathrm{~K}$, whilst the triple point was found at $T_{t}=1880 \mathrm{~K}$, supporting the claim that the model $\mathrm{C}_{60}$ has a thermodynamically stable liquid phase.

We have made no systematic investigation for the finitesize effect on the location of the critical point, which requires substantial amount of computer time. For this problem we refer to the previous GEMC study for $C_{60}$ which has shown that both $T_{c}$ and $\rho_{c}$ have a tendency to become higher with increasing particle number in the simulation box, ${ }^{7}$ which is in contradiction to the present nonsystematic analysis. We also note that the present free-energy analysis seems to suffer much less from the finite-size effect.

The final point to note is the experimental feasibility of testing simulations and theoretical calculations for the model $\mathrm{C}_{60}$. The recent $a b$ initio $\mathrm{MD}$ simulations ${ }^{31}$ have predicted that an isolated $\mathrm{C}_{60}$ molecule is stable against fragmentation up to $\sim 4500 \mathrm{~K}$ and a model of rigid $\mathrm{C}_{60}$ could be valid in the low-density vapor phase. However, experiments suggest that $\mathrm{C}_{60}$ molecules in the solid phase are unstable against polymerization $^{32}$ or decompose into an amorphous carbon ${ }^{33-35}$ well below the temperatures $(\sim 2000 \mathrm{~K})$ of interest. These results prevent us to think that the present and previous studies on the phase behavior of $\mathrm{C}_{60}$ are of partical importance. Nevertheless, this model itself is theoretically quite interesting as a typical example showing a unusual phase behavior which is an intermediate between those of ordinary atomic substances and systems of mesoscopic particles such as colloids.

\section{ACKNOWLEDGMENTS}

This work has been financially supported by the Grantin-Aid for Scientific Research under Grant No. 10640360. Part of this work was carried out under the Visting Researchers Program of the Institute for Materials Research (IMR), Tohoku University. We would like to thank the Computer Center of Iwate University and the HITAC S-3800/380 supercomputing facilities of the IMR for the continuous support. 
'A. P. Gast, C. K. Hall, and W. B. Russel, J. Colloid Interface Sci. 96, 251 (1983).

${ }^{2}$ N. W. Ashcroft, Europhys. Lett. 16, 355 (1991); Nature (London) 365, 387 (1993).

${ }^{3}$ M. H. J. Hagen, E. J. Meijer, G. C. A. M. Mooij, D. Frenkel, and H. N. W. Lekkerkerker, Nature (London) 365, 425 (1993).

${ }^{4}$ A. Cheng, M. L. Klein, and C. Caccamo, Phys. Rev. Lett. 71, 1200 (1993).

${ }^{5}$ L. A. Girifalco, J. Phys. Chem. 96, 858 (1992).

${ }^{6}$ M. Hasegawa and K. Ohno, J. Phys.: Condens. Matter 9, 3361 (1997).

${ }^{7}$ C. Caccamo, D. Costa, and A. Fucile, J. Chem. Phys. 106, 255 (1997).

${ }^{8}$ J. Q. Broughton, J. V. Lill, and J. K. Johnson, Phys. Rev. B 55, 2808 (1997).

${ }^{9}$ C. Caccamo, Phys. Rev. B 51, 3387 (1995).

${ }^{10}$ M. Hasegawa and K. Ohno, Phys. Rev. E 54, 3928 (1996).

${ }^{11} \mathrm{O}$. Umiguchi and $\mathrm{M}$. Hasegawa (unpublished).

${ }^{12}$ L. Mederos and G. Navascués, Phys. Rev. B 50, 1301 (1994).

${ }^{13}$ See, e.g., C. Caccamo, Phys. Rep. 274, 1 (1996).

${ }^{14}$ See, e.g., R. Evans, in Fundamentals of Inhomogeneous Fluids, edited by D. Henderson (Dekker, New York, 1992), p. 85.

${ }^{15}$ A. R. Denton and N. W. Ashcroft, Phys. Rev. A 39, 4701 (1989).

${ }^{16}$ M. Hasegawa, J. Phys. Soc. Jpn. 62, 4316 (1993); 63, 2215 (1994).

${ }^{17}$ W. A. Curtin and N. W. Ashcroft, Phys. Rev. Lett. 26, 2775 (1986).

${ }^{18}$ M. Hasegawa, J. Chem. Phys. 108, 208 (1998).

${ }^{19}$ M. Tau, A. Parola, D. Pini, and L. Reatto, Phys. Rev. E 52, 2644 (1995).
${ }^{20}$ See, e.g., Y. Tang, J. Chem. Phys. 109, 5935 (1998), and references therein.

${ }^{21}$ M. H. Hagen and D. Frenkel, J. Chem. Phys. 101, 4093 (1994).

${ }^{22}$ C. F. Tejero, A. Daanoun, H. N. W. Lekkerkerker, and M. Baus, Phys. Rev. Lett. 73, 752 (1994); Phys. Rev. E 51, 558 (1995).

${ }^{23}$ P. Bolhuis and D. Frenkel, Phys. Rev. Lett. 72, 2211 (1994).

${ }^{24}$ P. Bolhuis, M. H. J. Hagen, and D. Frenkel, Phys. Rev. E 50, 4880 (1994).

${ }^{25}$ A. Daanoun, C. F. Tejero, and M. Baus, Phys. Rev. E 50, 2913 (1994).

${ }^{26}$ N. F. Camahan and K. E. Starling, J. Chem. Phys. 51, 635 (1969).

${ }^{27}$ J, P. Hansen and I. R. McDonald, Theory of Simple Liquids (Academic, London, 1986).

${ }^{28}$ See, e.g., D. Frenkel and B. Smit, Understanding Molecular Simulation (Academic, San Diego, 1996).

${ }^{29}$ C. Rascón, L. Mederos, and G. Navascués, Phys. Rev. E 53, 5698 (1996).

${ }^{30}$ M. C. Abramo and G. Coppolino, Phys. Rev. B 58, 2372 (1998).

${ }^{31}$ K. Ohno, Y. Maruyama, and Y. Kawazoe, Phys. Rev. B 53, 4078 (1996).

${ }^{32}$ A. M. Rao, P. Zhou, K. A. Wang, G. T. Hager, J. M. Holden, Y. Wang, W. T. Lee, X. X. Bi, P. G. Eklund, D. S. Cornett, M. A. Duncan, and I. J. Amster, Science 259, 955 (1993).

${ }^{33}$ C. S. Sundar, A. Bharathi, Y. Hariharan, J. Janaki, V. S. Sastry, and T. S. Radhakrishnan, Solid State Commun. 84, 823 (1992).

${ }^{34}$ S. D. Leifer, D. G. Goodwin, M. S. Anderson, and J. R. Anderson, Phys. Rev. B 51, 9973 (1995).

${ }^{35}$ M. R. Stetzer, P. A. Heiney, J. E. Fisher, and A. R. McGhie, Phys. Rev. B 55, 127 (1997). 